\title{
An Eight-Lane 7-Gb/s/pin Source Synchronous Single-Ended RX With Equalization and Far-End Crosstalk Cancellation for Backplane Channels
}

\author{
Cosimo Aprile ${ }^{\circledR}$, Student Member, IEEE, Alessandro Cevrero, Member, IEEE, \\ Pier Andrea Francese, Senior Member, IEEE, Christian Menolfi, Member, IEEE, Matthias Braendli, \\ Marcel Kossel, Senior Member, IEEE, Thomas Morf, Senior Member, IEEE, \\ Lukas Kull, Senior Member, IEEE, Ilter Oezkaya ${ }^{\circledR}$, Student Member, IEEE, \\ Yusuf Leblebici, Fellow, IEEE, Volkan Cevher, Senior Member, IEEE, \\ and Thomas Toifl, Senior Member, IEEE
}

\begin{abstract}
This paper presents a versatile crosstalk cancellation scheme for single-ended multi-lane backplane links. Systemlevel investigations show that a scheme, which combines analog filters and decision-feedback crosstalk compensation on the receiver $(\mathrm{RX})$ side only, can efficiently remove crosstalk patterns in straight channels as well as boards with reflections due to via stubs. An eight-lane single-ended RX has been manufactured in 32-nm SOI CMOS to validate our findings. A CTLE and eighttap decision feedback equalizer equalize the channel without transmitter feedforward equalizer. A continuous time crosstalk canceller reduces precursors by nearest neighbors, while the residual postcursors from all aggressors are suppressed by direct feedback 7 × 8-tap decision-feedback crosstalk canceller (DFXC). Measurements with flip-chip packaged $R X$ show that the $R X$ macro can equalize both a 30-dB insertion loss single-ended channel with 0 -dB signal-to-crosstalk at Nyquist and a channel with 28-dB attenuation with the signal-to-crosstalk ratio of $6 \mathrm{~dB}$ combined with reflections due to via stubs. The $R X$ operates up to $7 \mathrm{~Gb} / \mathrm{s} /$ pin with PRBS11 data at bit error rate $(\mathrm{BER})<10^{-12}$, and occupies $300 \times 350 \mu \mathrm{m}^{2}$ with an energy efficiency of $5.9 \mathrm{~mW} / \mathrm{Gb} / \mathrm{s}$ from 1-V supply.
\end{abstract}

Index Terms-Continuous time linear equalizer, decisionfeedback equalizer, far-end crosstalk (FEXT), inter-symbol interference (ISI), source-synchronous architecture.

\section{INTRODUCTION}

A DVANCES in CMOS process technologies have led to an exponential increase in the digital processing power of high-performance microprocessor units, leading to an increment of the data transfer bandwidth between local chips.

Manuscript received July 7, 2017; revised October 11, 2017 and December 1, 2017; accepted December 2, 2017. This paper was approved by Guest Editor Jack Kenney. This work was supported in part by the European Commission through the ERC Future Proof and ERC Time-Data Tradeoffs and in part by the Swiss Science Foundation under Grant SNF 200021-146750 and Grant SNF CRSII2-147633. (Cosimo Aprile and Alessandro Cevrero contributed equally to this work.) (Corresponding author: Cosimo Aprile.)

C. Aprile, Y. Leblebici, and V. Cevher are with EPFL-STI-IEL-Lions/LSM, École Polytechnique Fédérale de Lausanne, CH-1015 Lausanne, Switzerland (e-mail: cosimo.aprile@epfl.ch).

A. Cevrero, P. A. Francese, C. Menolfi, M. Braendli, M. Kossel, T. Morf, L. Kull, I. Oezkaya, and T. Toifl are with the IBM Research Zurich, 8803 Rüschlikon, Switzerland.

Color versions of one or more of the figures in this paper are available online at http://ieeexplore.ieee.org.

Digital Object Identifier 10.1109/JSSC.2017.2783679



(a)

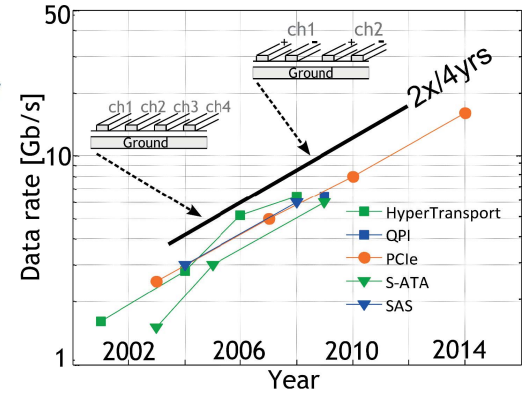

(b)
Fig. 1. (a) Backplane link, SE $4.8 \mathrm{~Gb} / \mathrm{s}$ [3]. (b) Pin data rate evolution across most common I/O standards [1].

Over the past years, the data rate required for each pin has almost doubled every four years across different I/O standards, as depicted in Fig. 1 [1]. However, due to packaging constraints as well as chip size limitation, the number of package pins is increasing only slightly, while the number of transistors served by one I/O approximately doubles every new CMOS technology node [2]. At the same time, low power consumption is a first-order design constraint for $\mathrm{I} / \mathrm{O}$ circuits. ITRS assumes that high-performance serial transceivers can consume a maximum of $10 \%$ of the chip power and I/O links should occupy maximum $20 \%$ of the entire chip area.

In combination with innovative circuits techniques, adopting single-ended signaling technology doubles the performance (bandwidth per pin) with respect to similar channel boards operating with differential lines per signal, such as Quick Pack Interconnect by Intel [4] and Hypertransport by AMD [5]. The main limitation of using single-ended printed circuit board (PCB) traces comes from the increase in crosstalk (XTC) noise due to electromagnetic coupling because of increased wire density. As data rate increases, crosstalk becomes then the most significant noise source in single-ended parallel links.

Crosstalk is the combination of far-end and near-end electromagnetic coupling. near-end crosstalk does not affect the signal integrity in unidirectional links [6], while far-end crosstalk (FEXT) heavily affects single-ended PCB traces. Conventionally, board-level techniques allow to handle FEXT, 
increasing, for instance, the distance between channels, or including shielding techniques [7], [8]. However, these techniques require additional space on PCB and are rarely implemented in high-density and high-speed links. On the circuit side, there is a lack of crosstalk cancellation schemes that simultaneously handle a multichannel board. Most previous works focus on crosstalk compensation circuits for memory channels. Crosstalk-induced timing distortion is reduced by means of the timing-delay adjustment of data transition versus the state of the data [9]. However, the challenge is in knowing the correct timing compensation, which is also dependent on the process variation [10]. Crosstalk in the memory interface has also been addressed by Bae et al. [11], where it limits the maximum number of transitioning lanes, but does not compensate for the distorted signals. Other approaches to compensate for crosstalk noise include the use of staggered I/Os combined with a glitch suppression scheme to improve vertical eye opening or a slew rate control driver on transmitter (TX). Sham et al. [12] proposed to cancel FEXT injected by neighboring aggressor lanes by using finite impulse response at the TX. Nazari and Emami-Neyestanak [13] used a switched capacitor technique linearly combining two analog signals to reduce crosstalk, where the amount of FEXT is controlled attenuating a passive filter output.

All of the previous designs do not address the multiple crosstalk lane sources per channel bundle. To the best of our knowledge, for the first time, Oh and Harjani [14] adequately addressed crosstalk issues considering a minimum of four lanes for single-ended channels. The receiver (RX) macro proposed by Oh and Harjani [14] is power efficient and can be extended to an infinite number of lanes. However, it trades FEXT compensation by the increase of wire spacing every two lanes to minimize residual error terms. In addition, the channel used for measurement has a relatively low insertion loss $(-11 \mathrm{~dB}$ at Nyquist frequency) and does not requires power hungry decision feedback equalizer (DFE) function. All of the proposed schemes have poor adaptability to different FEXT environments or result large circuit complexity or significant power consumption.

In this paper, we propose a versatile RX circuit capable of coping with large insertion loss and can minimize crosstalk with multiple channels having the identical lane spacing and important channel attenuation of 28 and $30 \mathrm{~dB}$ at Nyquist frequency. This paper extends our previous work [15], by considering different crosstalk reduction methodologies tailored with the channel board characteristics (see Section II-B). Furthermore, we provide more electrical characterizations (see Section IV), which are aligned with the system-level analysis (see Section II) and demonstrate the inter-symbol interference (ISI) equalization and crosstalk reduction strength of the overall RX circuit. This paper is organized as follows. The system-level analysis is discussed in Section II, while the RX macroarchitecture and its functional units are described in Section III. Section IV gives the electrical measurements and Section V discusses the results and concludes this paper.

\section{SYSTEM-LEVEL ANALYSIS}

In high-loss single-ended communication links, the main signal path needs to be equalized to cope with ISI noise.

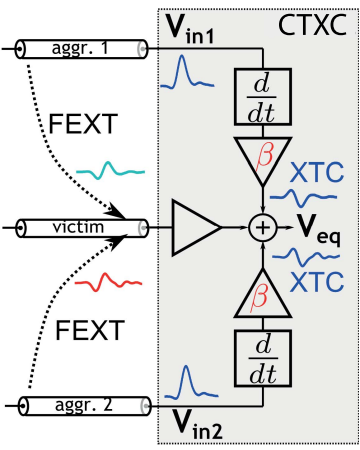

Fig. 2. Crosstalk cancellation using CTXC front end on three-lane channel.

Moreover, the precursors, cursor, and postcursors FEXT components need to be minimized to improve the signal integrity.

In this section, we first discuss the crosstalk cancellation strategy we adopt on the RX macro (see Section II-A), and then, we present the different channel board characteristics used to test the chip (see Section II-B). The mathematical paragraph (see Section II-C) discusses the crosstalk reduction in ideally coupled lanes, highlighting its limitations. Section II-D shows the system-level simulations of a continuous time crosstalk canceller (CTXC) and a decision feedback-based crosstalk canceller (DFXC) blocks, highlighting how their coexistence has to be tailored depending on the channel board characteristics. The last part of this section (see Section II-E) analyses the crosstalk cancellation techniques proposed in this paper, in case of skewed board lanes.

\section{A. Crosstalk Cancellation Considerations}

Analog filters can be used at the RX side to remove crosstalk components. The compensation scheme relies on the fact that, in ideally coupled lanes, FEXT is proportional to the derivative of the crosstalk source signal. A differentiation (easily implemented with analog filters), with appropriate gain $\beta$, can then reproduce FEXT and subtract it to the forward signal component to effectively remove FEXT [16]. It is possible to replace the RX analog filters with a feedforward equalizer at the transmitter (TX) side. However, such architecture is unable to prevent jitter amplification in the transmitted signal and imposes stringent linearity specifications in the output drivers.

For these motivations, in the proposed I/O link, the received data from adjacent lanes are processed in the analog domain by means of a CTXC, ${ }^{1}$ to generate precursors and cursor FEXT cancellation signals [14], [15], [17]. The CTXC concept for three-lane channel is shown in Fig. 2, where a passive differentiator block is used to emulate the FEXT signal.

In presence of an eight-lane single-ended bus, the extension of the scheme introduced for three-lane system would differentiate the received signals from the seven aggressors (the crosstalk sources) and add them to the forward signal lane with appropriate gain. However, processing the received signal from all aggressor lanes to remove FEXT in a defined victim lane (the crosstalk recipient) is not a practical solution,

\footnotetext{
${ }^{1}$ Named XCTLE in [15].
} 
TABLE I

Key PARAmeters of THE Crosstalk BoARd

\begin{tabular}{|c|c|c|c|}
\hline Name & Extensions \& Stubs & Length & Geometry \\
\hline Ch1 & none & $995 \mathrm{~mm}$ & $\mathrm{~s}=1.5 \times \mathrm{w}=142 \mu \mathrm{m}$ \\
\hline Ch2 & 2 via stubs & $720 \mathrm{~mm}$ & $\mathrm{~s}=1.5 \times \mathrm{w}=142 \mu \mathrm{m}$ \\
\hline
\end{tabular}

since it would enlarge the capacitance at the summation node, limiting the bandwidth of the overall system.

Based on these motivations, our CTXC implementation receives the signals from the two adjacent channels only, which have the greatest impact on the signal integrity over the victim lane. By doing so, large FEXT cursor and precursor components can be reduced.

The residual crosstalk noise is then treated and effectively removed by means of a decision-feedback based block, crossconnected between each lane of the channel board [15], [17]. The analog correction of such DFXC, ${ }^{2}$ is based on the switchcap approach proposed in [18]. As a result, the transmitted signal over each lane is corrected by CTLE and DFE for the ISI distortion, while CTXC and seven DFXCs minimize the crosstalk noise.

The CTXC and DFXC systems dovetail and ensure the crosstalk cancellation completely on the RX side, thereby coupling the RX circuit with TXs sourced by different vendors.

\section{B. Boards Characteristics}

In this paper, two different boards have been used to emulate multi-lane single-ended legacy channels for servers' applications. For consistency, we consider two complementary cases, (their main parameters are in highlighted in Table I), labeled $\mathrm{Ch} 1$ and $\mathrm{Ch} 2$, which are defined as follows.

1) Ch1 Board: The crosstalk board Ch1 consists of a Rogers-PCB mother card, which hosts eight clean channels (no notches in the frequency response) due to the absence of extension boards, vias, and connectors. The signal travels for $995 \mathrm{~mm}$ on the mother card, in a lane defined by its trace width $w=95 \mu \mathrm{m}$ and lane-to-lane spacing $s=142 \mu \mathrm{m}$. Ch1 is an example of large attenuation channel, $-30 \mathrm{~dB}$ at Nyquist frequency, and important FEXT contribution. Fig. 3(a) displays the S-parameters (insertion loss and FEXT from all switching lanes) with respect to lane 3, in each channel bundle.

2) Ch2 Board: Channel Ch2 consists of a $720-\mathrm{mm}$ RogersPCB mother card with extension Rogers-PCB board mounted on top with two Erni MicroSpeed connectors. The signal travels for $100 \mathrm{~mm}$ on the mother card, then goes to the first extension board, travels back to the mother card for $100 \mathrm{~mm}$, travels in the second extension board, and finally arrives to the RX. In this channel, $s=1.5 \times w=142 \mu \mathrm{m}$. Board Ch2 FEXT does not follow the ideal derivative model, due to the presence of connectors and via arrays in the signal path. With respect to $\mathrm{Ch} 1, \mathrm{Ch} 2$ results as a more severe board channel, with an important channel attenuation around $28 \mathrm{~dB}$ and a more severe FEXT contribution. Fig. 3(b) displays the S-parameters for lane 3 in each channel bundle.

\footnotetext{
${ }^{2}$ Named XDFE in [15].
}

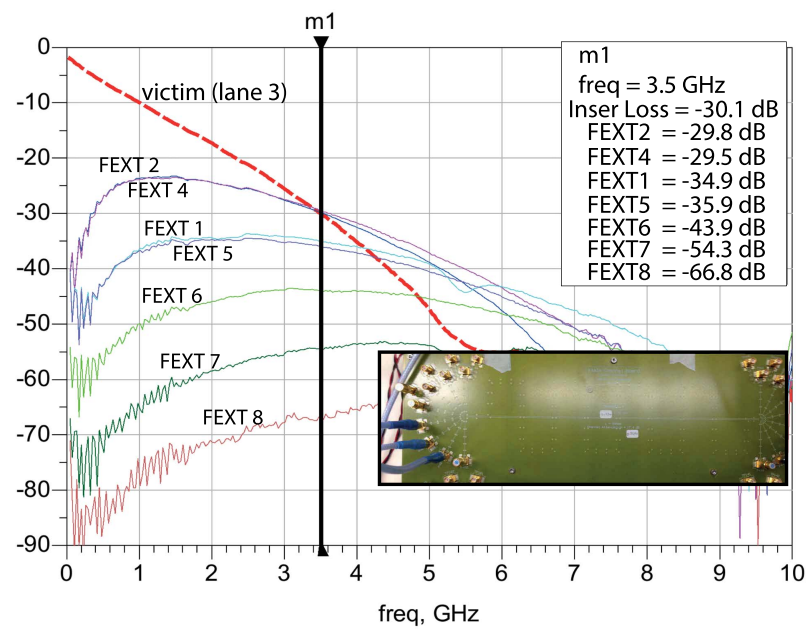

(a)

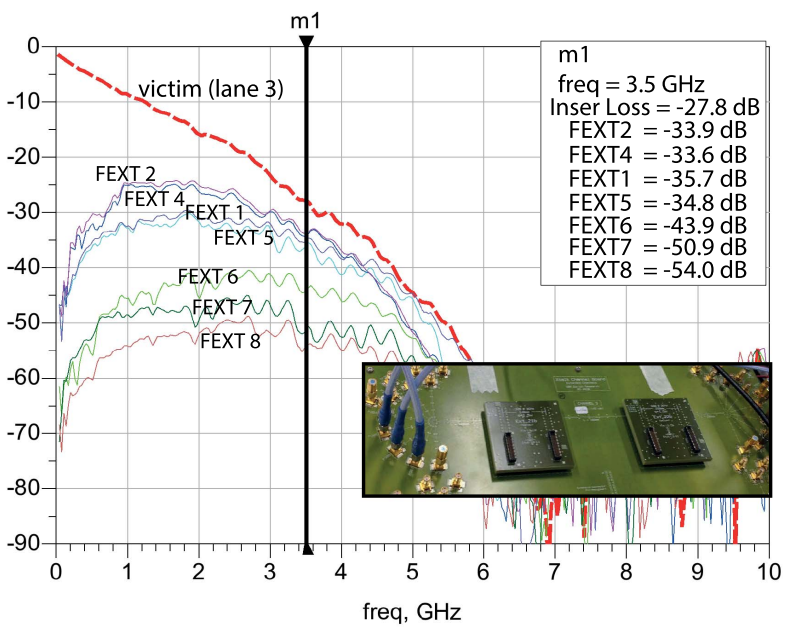

(b)

Fig. 3. Forward and FEXT frequency responses (magnitude) for (a) $\mathrm{Ch} 1$ and (b) $\mathrm{Ch} 2 \mathrm{PCB}$ board.

\section{Mathematical Formulation for Ideally Coupled Lanes}

This section provides an overview of the crosstalk contribution within $N$ ideally coupled lanes (e.g., Ch1 board), where the FEXT follows the derivative model [16].

The frequency-domain representation of the received vector signal $\mathbf{Z} \in \mathbb{R}^{N}$ is given by $\mathbf{Z}=\mathbf{G} \cdot \mathbf{H} \cdot \mathbf{X}$ [14], where $\mathbf{G} \in$ $\mathbb{R}^{N \times N}$ is given by the CTXC contribution, $\mathbf{H} \in \mathbb{R}^{N \times N}$ is the frequency channel response matrix, and $\mathbf{X} \in \mathbb{R}^{N}$ is the input signal vector.

As addressed in [14], the setting that ensures zero crosstalk contribution from the nearest neighbor is $G_{x}=\beta G_{0}$, where $G_{x}$ and $G_{0}$ define the CTXC analog gain for the crosstalk cancellation component and forward received strength, respectively. Under this scenario, the multiplication between the channel response matrix $\mathbf{H}$ and the CTXC matrix $\mathbf{G}$ shows the additional reused crosstalk energy $\left(2 \omega^{2} \beta^{2}\right) G_{0} H$. Nonetheless, it also shows an error contribution at each lane from the second neighbors and reveals the presence of additional uncompensated noise terms $\omega^{2} \beta^{2} G_{0} H$. In [14], it is proposed to solve this issue by pairing up every two lanes and maintaining sufficient distance between the bundle, thereby trading board 


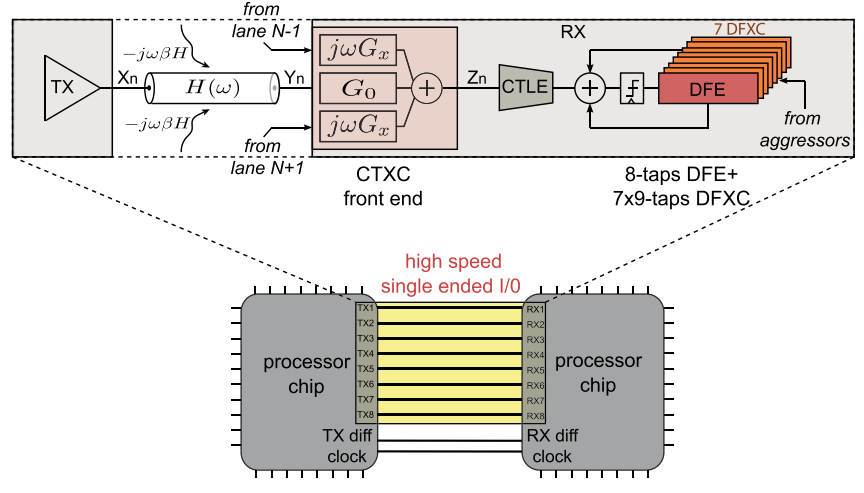

Fig. 4. Single-lane transceiver block diagram with crosstalk compensation scheme combining CTXC on the front end.

area for residual error term. However, this reduces the PCB area efficiency, and it may not even be possible in applications where dense PCB routing is required. It should be noticed that using such an analog front end, residual errors' terms can never be forced to zero.

In this paper, instead of zero forcing the FEXT from adjacent lanes (setting $G_{x}=\beta G_{0}$ ) and trying to minimize the error term by increased board spacing, we optimize the gain settings $G_{x}$ and $G_{0}$ in the CTXC with the goal of maximizing the vertical and horizontal eye opening. Paragraph II-D1 discusses this crosstalk reduction technique, applied for channel Ch1, where CTXC is sufficient to open the eye diagram in each lane.

However, if the board presents connectors and via arrays in the signal path (e.g., Ch2 board), the crosstalk patterns will be more intricate and will not follow the ideal coupled lanes model. Therefore, the CTXC only would not be sufficient to ensure operations at BER $=10^{-12}$, and necessitates the DFXC to reduce the FEXT postcursors. Such a crosstalk cancellation technique is addressed in Section II-D2.

\section{System-Level Simulations}

A system-level analysis is performed to investigate the optimized crosstalk cancellation strategies for the channels described in Section II-B.

Fig. 4 highlights one of the eight single-ended lanes within the channel bundle. The eight-lane channel bundle frequencydomain data (forward and FEXT response) have been collected in a 16-port S-parameter file, which models the interconnect. On the RX side, each lane features a CTXC followed by a CTLE, 8-tap DFE and $7 \times 8$-tap DFXC. In the eight-lane topology, it is assumed that data patterns of different lanes are uncorrelated.

1) Ch1 Board Crosstalk Reduction: In this section, the crosstalk reduction technique applied for Ch1 board (described in Section II-B1) is addressed.

The calibration is performed over $R, C, G_{x}, G_{o}$, and CTLE settings. Due to large channel attenuation, both CTLE and DFE are required to effectively remove ISI. The simulation results are presented in Fig. 5. All simulations include a 5-ps TX random uncorrelated jitter (roughly $3.5 \%$ UI for $7-\mathrm{Gb} / \mathrm{s}$

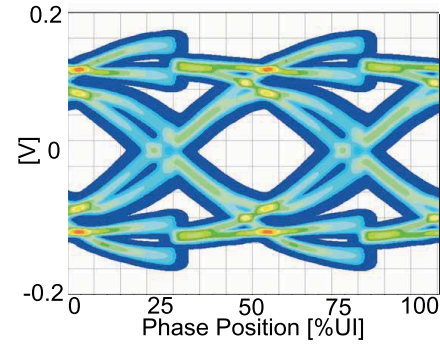

(a)

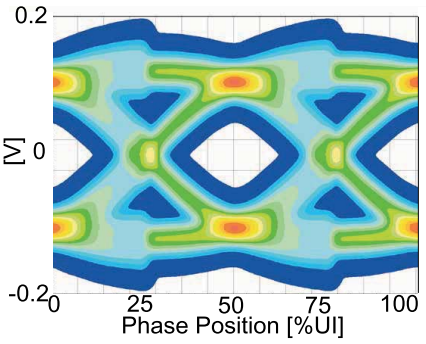

(c)

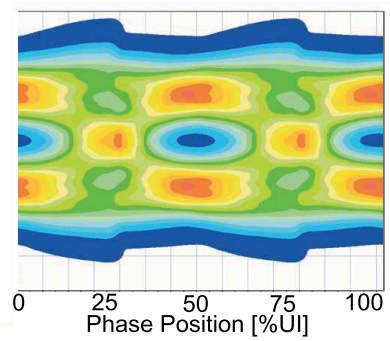

(b)

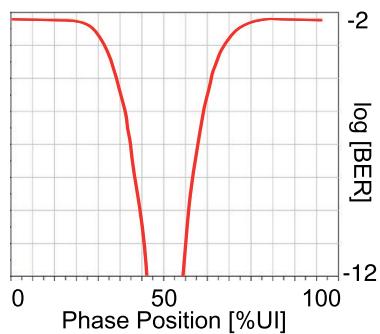

(d)
Fig. 5. Simulated RX data eye for $\mathrm{Ch} 1$ board, with all aggressors (a) switched off and (b) switched on without crosstalk compensation scheme (CTLE and DFE on, in both cases). (c) Data eye and (d) bathtub plot with optimally calibrated CTXC front end. All aggressors are transmitting.

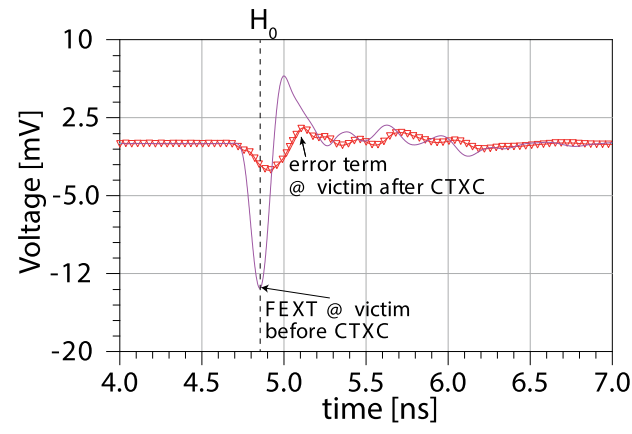

Fig. 6. FEXT pulse response from the aggressor to victim lane before and after CTXC.

data rate). The data eye is completely closed when all aggressors are transmitting. The CTXC front end is able to open a closed data eye with 35\% UI eye width and $75-\mathrm{mV}$ eye height. Fig. 6 shows the FEXT pulse response between the aggressor and victim at $7 \mathrm{~Gb} / \mathrm{s}$, before and after the crosstalk compensation scheme (with optimal filter setting's calibration). Such an analysis highlights that the CTXC makes the system less sensitive to jitter noise, since it flattens the derivative of the crosstalk pulse response.

2) Ch2 Board Crosstalk Reduction: This section discusses the crosstalk reduction technique addressed for channel board $\mathrm{Ch} 2$ (defined in Section II-B2). The limits of CTXC with this particular board are evinced in Fig. 7, which shows a completely closed eye for $7 \mathrm{~Gb} / \mathrm{s}$ with only the twonearest aggressor lane switching. Thus, a different crosstalk minimization technique is involved for this type of channel board. First, the CTXC-CTLE strength calibration targets the reduction of precursors and cursor crosstalk contribution. Then, the crosstalk terms are determined from detected bits in the aggressor lanes and its derived voltage is subtracted over the victim by means of the DFXC filters. A statistical 


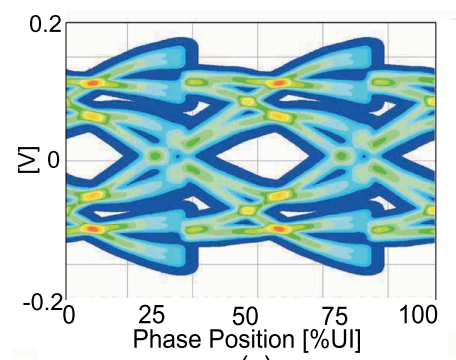

(a)

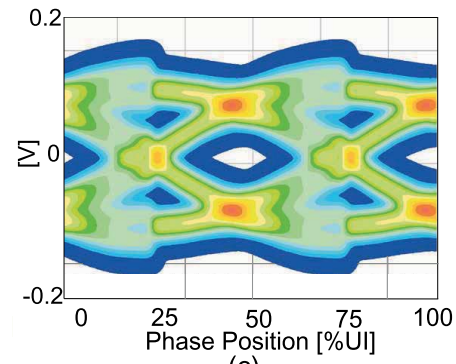

(c)

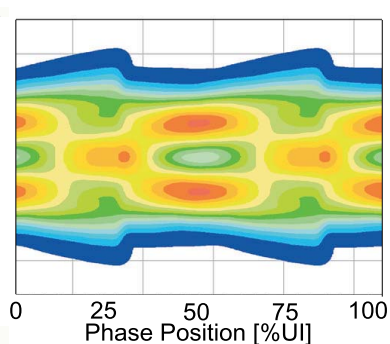

(b)

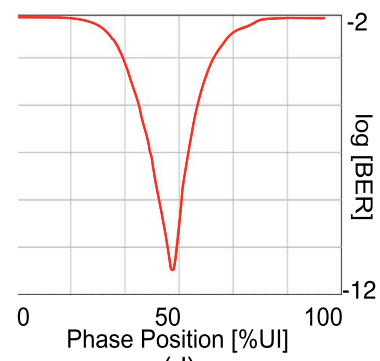

(d)
Fig. 7. Simulated RX data eye for $\mathrm{Ch} 2$ board, with all aggressors (a) switched off and (b) switched on without crosstalk compensation (CTLE and DFE on, in both cases). (c) Data eye and (d) bathtub plot with optimally calibrated CTXC front end with the two nearest aggressors transmitting.

analysis using MATLAB Software has been performed to validate the CTXC-DFXC effect. A probability distribution function (pdf) of the ISI and crosstalk pulse response spanned over all postcursor taps has been developed, where values have been found convolving the ISI and crosstalk terms. The crosstalk pdf allows to analyze all the possible combinations of postcursor ISI and the FEXT taps. Such an analysis is valid by assuming that the data over the victim and the aggressors are white and uncorrelated.

To perform such analysis, the pulse response on the victim lane (only the victim lane TX is transmitting, while all the aggressors are silent) is combined with the crosstalk pulse responses (only the aggressor transmits a pulse, while the victim TX is silent) of the aggressor lanes. During this phase, the CTLE is activated, while DFE is OFF. Given a sampling window $\mathcal{D}=\left\{x_{1}, \ldots, x_{m}\right\}$ of $m=32$ sampling points in $1 \mathrm{UI}$, the optimal $h_{0}$ has been found by choosing the index of the sampling point in $\mathcal{D}$ that maximizes the eye aperture $V_{\text {eye }}$, which is defined as

$$
V_{\text {eye }}=\text { Signal }-\mathrm{CDF}_{\mathrm{NIX}}^{-1}-\text { Sensitivity }
$$

where Signal $=h_{0}-\left|h_{-1}\right|$ is the signal amplitude given by the cursor $h_{0}$ minus the absolute value of the first of the precursors $h_{-1}$ (which has the same polarity as the cursor value in these types of channels); $\mathrm{CDF}_{\text {NIX }}^{-1}$ is the inverse of the cumulative distribution function (CDF) of the noise, ISI, and crosstalk (NIX) at BER $=10^{-12}$. The CDF is computed convolving the ISI, crosstalk, and noise distributions, given by the pdf analysis. The input referred noise includes CTLE, comparator, and jitter noise. The term Sensitivity $=5 \mathrm{mV}$ is the minimum comparator voltage sensitivity.

Using the precise pdf approach for analyzing ISI and crosstalk has been necessary, since using a simpler rms summation of ISI and crosstalk components was found to give


Fig. 8. Highlight of (a) vertical eye aperture and (b) signal, crosstalk, and ISI evolution for different CTLE peaking settings.
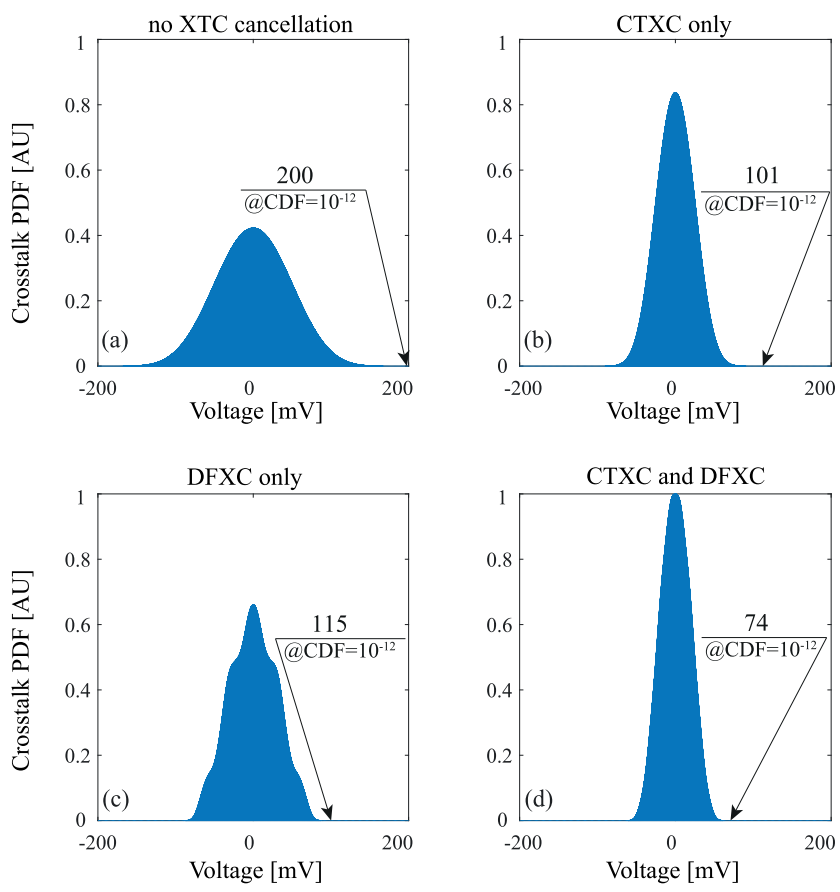

Fig. 9. PDF of the crosstalk pulse response spanned over all postcursor taps (a) without crosstalk cancellation, (b) with only CTXC on, (c) with CTXC off and DFXC on, and (d) with both CTXC-DFXC activated.

overly pessimistic results, which is due to the non-Gaussian nature of distributions for ISI and crosstalk.

Fig. 8(a) shows the vertical eye opening versus CTLE peaking settings, including 8-tap DFE equalization co-optimized with CTXC and 56-tap DFXC at BER $=10^{-12}$. The trend reveals that high peaking settings provide the maximum vertical eye opening, even in the presence of crosstalk. This counter intuitive trend can be explained in Fig. 8(b), where the signal, ISI, and crosstalk contribution (derived by the pdf analysis) are plotted independently. Eventhough the CTLE peaking is generated by lowering the dc gain, larger CTLE peaking settings increase the signal. This is because lower peaking results in more ISI, which requires the CTLE output to be scaled to meet linearity requirements for DFE equalization. Moreover, crosstalk components increase with CTLE peaking, since the CTLE tends to amplify crosstalk. Overall, increasing the CTLE peaking, the signal grows faster than crosstalk does and ISI is reduced; then, the net eye opening is larger with high peaking settings.

The crosstalk pdf obtained with the statistical analysis is reported in Fig. 9 in four different scenarios, with the maximum CTLE peaking setting. In particular, Fig. 9(a) shows the 
TABLE II

Crosstalk Cancellation Performances

\begin{tabular}{|c|c|c|c|c|}
\hline \multicolumn{5}{|c|}{ Board Ch2 } \\
\hline Signal $=h_{0}-h_{-1}[\mathrm{mV}]$ & \multicolumn{5}{|c|}{4} \\
\hline$\Delta_{\text {ISI }}[\mathrm{mV}]$ & \multicolumn{5}{|c|}{5} \\
\hline$\sigma_{\text {Noise }}[\mathrm{mV}]$ & \multicolumn{5}{|c|}{} \\
\hline Sensitivity $[\mathrm{mV}]$ & OFF & ON & OFF & ON \\
\hline CTXC & OFF & OFF & ON & ON \\
\hline DFXC & 200 & 101 & 115 & 74 \\
\hline$\Delta_{X T K}[\mathrm{mV}]$ & 211 & 114 & 126 & 88 \\
\hline$\Delta_{I S I+X T K}[\mathrm{mV}]$ & 214 & 121 & 131 & 95 \\
\hline$\Delta_{I S I+X T K+N o i s e}[\mathrm{mV}]$ & 214 & -7 & 2 & 38 \\
\hline$V_{\text {eye }}[\mathrm{mV}]$ & -105 & -7 & & \\
\hline
\end{tabular}


Fig. 10. (a) Skewed and (b) un-skewed impulse responses at the TX side.

crosstalk pdf with no FEXT cancellation, while in Fig. 9(b), only the CTXC is activated, reducing the crosstalk noise amplitude at BER $=10^{-12}$ from 200 to $113 \mathrm{mV}$. The pdf distribution for the DFXC-only is reported in Fig. 9(c). When CTXC is combined with DFXC, as shown in Fig. 9(d), the crosstalk error term $\Delta_{\mathrm{XTK}}$ at $\mathrm{BER}=10^{-12}$ is equal to $73.5 \mathrm{mV}$, showing a significant improvement in vertical eye opening. The results from the pdf analysis are reported in Table II, which highlights the crosstalk cancellation strength for all the CTXC-DFXC combinations. Without crosstalk reduction, the FEXT contribution overcomes the cursor $h_{0}$ amplitude, resulting to a closed eye. The vertical eye aperture is improved, once both the CTXC and the DFXC crosstalk canceller blocks are optimally calibrated, resulting in $38.7-\mathrm{mV}$ vertical eye opening.

\section{E. Crosstalk Cancellation Over Skewed Lanes}

Some differences in the lane length, due to manufacturing tolerance, can be the cause of some skew experienced by the non return to zero (NRZ) signal traveling the channel bundle, both on the TX and the RX side. Fig. 10(a) shows the skewed impulse responses for all the eight single-ended lanes of channel board $\mathrm{Ch} 2$, once the signals are launched at the same time at the TX. Such an unwanted issue can be solved forcing delay adjustments on the TX side, resulting in aligned impulse responses, as depicted in Fig. 10(b).

However, the skew adjustment on the TX for each forward paths does not solve the crosstalk pulse responses skew issue on the RX side. Fig. 11(a) shows two signals traveling a multi-lane board with identical channel lengths. The crosstalk coupling from the aggressor to the victim lane is then perfectly corrected by the CTXC signal, given by $-\beta d / d t$ of the aggressor pulse response, at the time $t_{0}$.



Fig. 11. Qualitative highlight of CTXC effects for (a) un-skewed and (b) skewed board lanes.

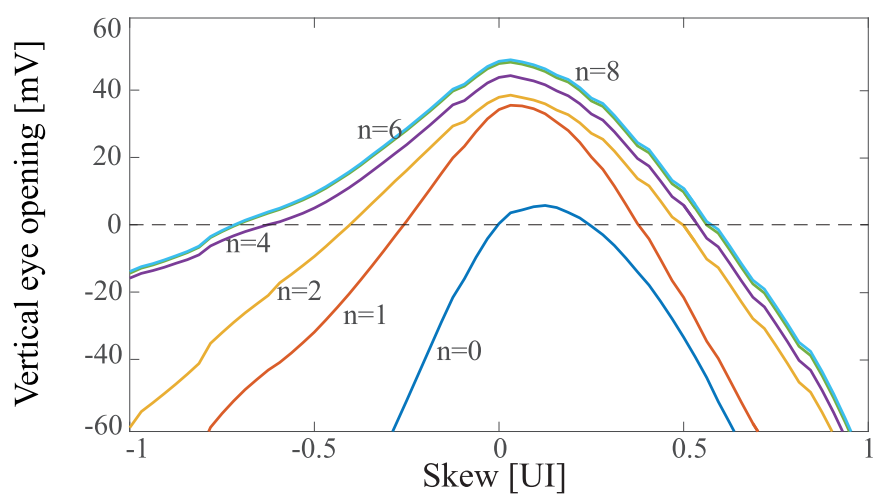

Fig. 12. Vertical eye aperture versus different lane skews at the RX side, with a different number $n$ of taps activated on the DFXC. The simulations are performed with the $\mathrm{Ch} 2$ channel board.

In case of different channel lengths, the crosstalk coupling signal arrives to the RX terminal at a different instant with respect to its correction version because of the channel skew. Considering that the aggressor lane is shorter than the victim lane, as highlighted in Fig. 11(b), the XTC coupling arrives at time $t_{0}-t_{\text {skew }}$, while the FEXT cancellation signal, generated in the CTXC, is ready at time $t_{0}$. This produces a residual crosstalk signal, which might be partially reduced by the DFXC. For this reason, the CTXC of each lane has to be adapted accordingly.

An analysis is performed to verify how the DFXC system interacts with the RX system sensitivity. Fig. 12 shows the vertical eye aperture with different lane skews at the RX, with a different DFXC number of taps activated, over a single lane of Ch2 board. The DFXC contribution is already evident on the vertical eye aperture, from no crosstalk reduction (i.e., $n=0$ curve) to tap- 1 of the DFXC activated ( $n=1$ curve). Interestingly, it is important to evince how the DFXC reduces the sensitivity to the skew. For instance, considering in Fig. 12 


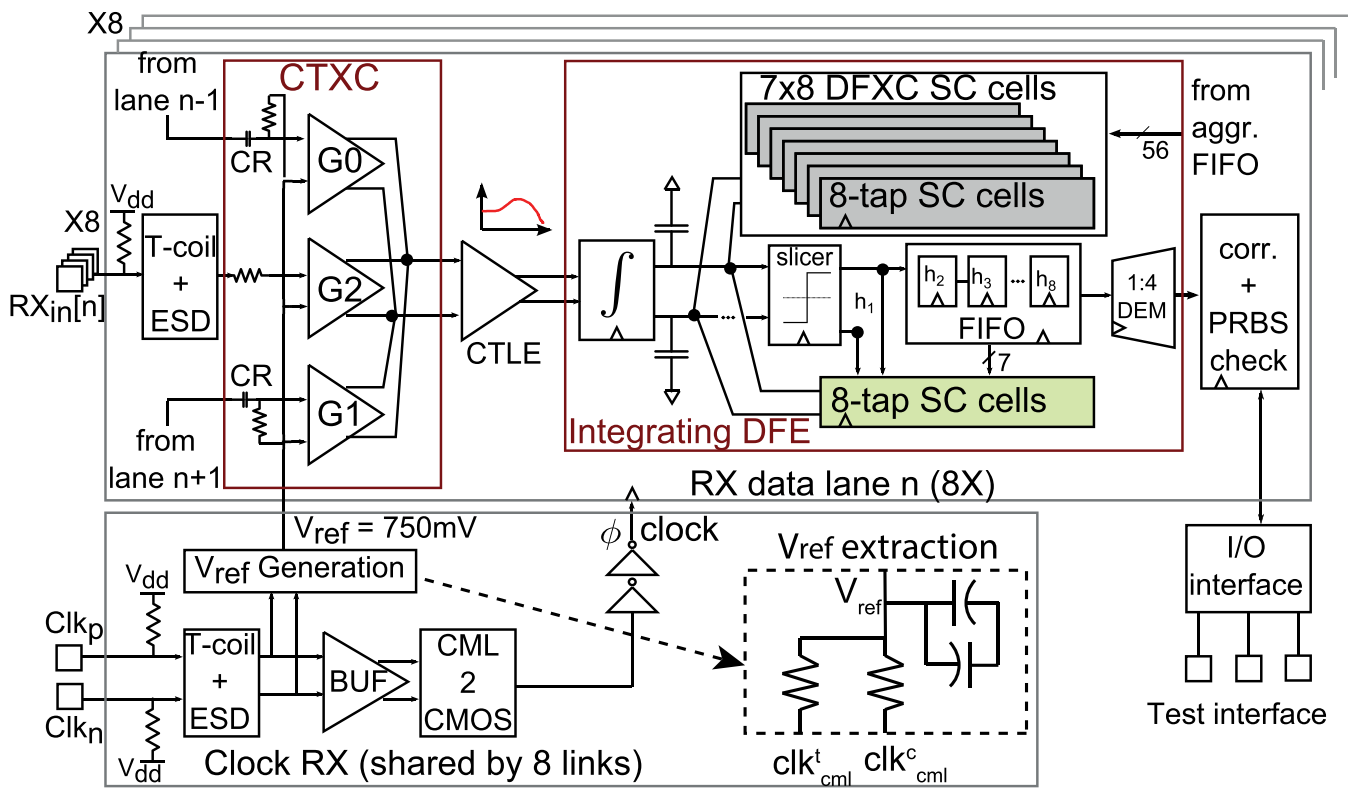

Fig. 13. Eight-lane single-ended RX architecture.

the curve with the first four taps activated (i.e., $n=4$ curve), the vertical eye opening is flattened with respect to the one without DFXC contribution. Moreover, the DFXC contribution is limited to the first $2-4$ taps, since only marginal crosstalk reduction is obtained with more taps activated.

\section{ReCEIVER ARChitecture AND CiRCUits}

The architecture of the source synchronous RX is shown in Fig. 13. It consists of eight single-ended data lanes and one shared differential clock lane. Each datapath starts with the termination front end, followed by a product-level electrostatic discharge (ESD) protection combined with T-COIL for bandwidth extension. The CTXC processes the input signal together with the nearest aggressor. The CTXC output goes to a two-stage CTLE followed by a direct feedback 8-tap DFE and 56-tap DFXC running at full rate. Equalized output at full rate is then deserialized to quarter rate and sampled by a digital engine, used for adaptation and BER check.

\section{A. Clock Generation}

A full rate clock supplied off chip with $1-\mathrm{V}_{\mathrm{pp}}$ swing and 750-mV CM is terminated differentially before being amplified by a CML buffer [19]. The reference voltage $V_{\text {ref }}$ is extracted directly from the input clock common mode without the need of a dedicated pin as in many single-ended standards, such as DDRX. The buffered input clock is then converted to CMOS level and buffered to the local clock distribution within each lane.

\section{B. CTXC and CTLE}

The CTXC is located after the impedance matching network and presents an FEXT reduced signal to the CTLE. Fig. 14 shows the circuit implementation of the proposed CTXC circuit.

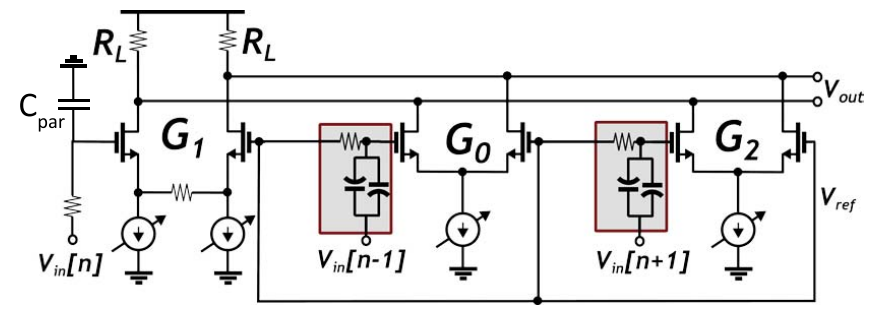

Fig. 14. CTXC stage with single-ended passive differentiator, variable gain amplifier, and current summation. The two high-pass $R C$ differentiators are highlighted in the boxes.

The CTXC consists of two passive differentiators followed by a current domain adder. The differentiators produce a single-ended crosstalk cancellation signal from the two adjacent lanes. The values of $R=972 \Omega$ and $C=30 \mathrm{fF}$ have been chosen to provide return loss below $-10 \mathrm{~dB}$ up to $4 \mathrm{GHz}$ at each of the broadband $50-\Omega \mathrm{RX}$ inputs. In [14], a resistor-capacitor replica circuit is added in the forward path to equalize phase delays between forward and crosstalk cancellation paths. In this way, the transfer function of the differentiator differs from the replica circuit by $s \mathrm{RC}$, providing $90^{\circ}$ phase shifts at all frequencies. However, this creates a parasitic pole on the main signal path. In this design, only the resistor is added in the main path, while the capacitor consists of the CTXC input stage loading directly. Circuit simulations across corners resulted in acceptable distortion with marginal impact on crosstalk cancellation.

A current domain adder with programmable gain combines the signals from the three paths. Three digitally programmed bias currents enable to adjust the gain of the forward and crosstalk cancellation paths independently. Variable gain amplifier (VGA) bias currents are binary weighted and can be adjusted with 4-bit resolution, enabling crosstalk cancellation over a wide range. The forward path uses a degenerated differential pair to improve linearity. Since the differentiated 




Fig. 15. Simulated ac response of main signal path VGA with maximum gain setting.

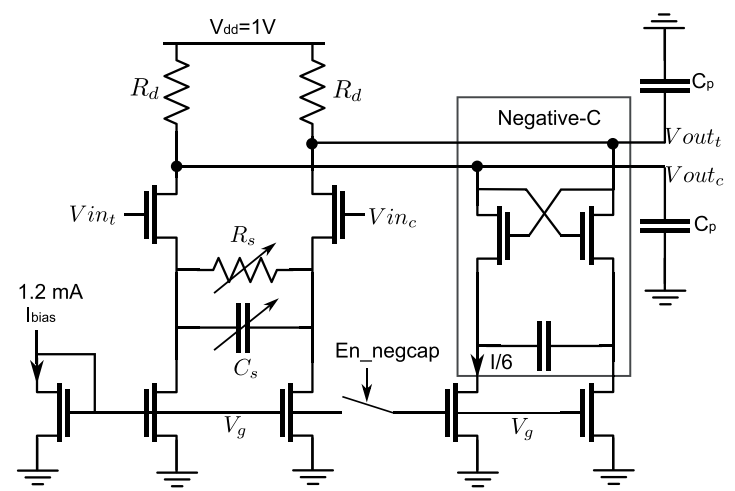

Fig. 16. CTLE stage with negative-C bandwidth enhancement. Reprinted from [18].

signals have a small amplitude, because FEXT is typically much smaller than the main signal component, there is no degeneration resistor in the crosstalk cancellation VGA. The single-ended to differential conversion is performed in the CTXC directly by connecting the VGA input differential pair to $V_{\text {ref }}$ on one side and to the differentiator/ compensator on the other side.

Fig. 15 displays the simulated (after $R C$ extraction) frequency response of the forward path VGA. The dc gain is $3.9 \mathrm{~dB}$ with a 3-dB bandwidth of $4.19 \mathrm{GHz}$. Bandwidth limitations come from the large capacitance at the current summation node, which corresponds to $16 \mathrm{fF}$. This is still acceptable for $7-8 \mathrm{~Gb} / \mathrm{s}$, and hence, no architecture change is needed.

The CTLE circuit, depicted in Fig. 16, is a differential buffer stage with programmable capacitive and resistive source degeneration [18]. A negative capacitance is in parallel with the differential pair and, if enabled, is used to enhance the bandwidth of the circuit. The programmable resistive degeneration is controlled with 9 thermometer coded steps, providing 17 settings in total. The degeneration capacitance is binary programmable with 4-bit resolution. Each capacitance step is implemented with two anti-parallel connected varactors. Two CTLE stages are cascaded to provide up to $17-\mathrm{dB}$ peaking at $3.5 \mathrm{GHz}$ with $-3.7 \mathrm{~dB}$ dc gain.

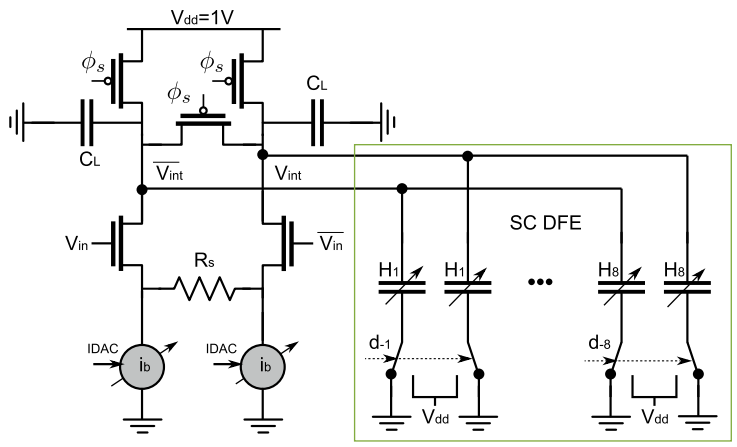

Fig. 17. Integrating DFE using SC feedback.

\section{DFE and DFXC}

The DFE core, shown in Fig 18, includes 8-tap DFE and $7 \times 8$ DFXC switched-capacitor cells. A current integrating stage amplifies the CTLE output for $1 / 2$ UI. A track and hold stage is avoided to limit the $\mathrm{kT} / \mathrm{C}$ noise with a cost of 0.9-dB loss due to half UI time window integration. The DFE core loop is based on a direct feedback full rate DFE, where the critical timing loop is for tap- $1\left(h_{1}\right)$ equalization feedback. Digitally programmable switched capacitor digitalanalog converters (SC-DAC) are implemented to add charge on the integration node. This approach enables a fast DFE feedback thanks to the instantaneous effect of the charge injection on the summation node and allows a relaxation of the DFE timing loop, compared with current summation DFE [18]. Each capacitive DAC is programmable with 6-bit resolution, with $1 \mathrm{LSB}=250 \mathrm{aF}\left(C_{\max }=15.75 \mathrm{fF}\right)$ and is realized with metal M1 and M2 layers for the finger caps. Correction tap $h_{1}$ uses three SC cells connected in parallel, allowing a wider range correction. The less critical DFE taps $h_{2}$ to $h_{8}$ and the remaining 48-DFXC taps are driven by FIFO data. The implemented DFE, with a capacitance charge feedback, is shown in Fig. 17. A dynamic differential latch receives the digital data resolved by the strongARM data-latch [18], and samples them at the falling clock edge. The differential cascoded voltage switch (DCVS) and dynamic latch together implement the function of a flip-flop. The dynamic latch avoids charge injection, which occurs before the integration period. In fact, in an SC-DFE, no DFE correction is performed if the charge injection occurs during the reset phase. The data format is kept in pre-charged dynamic logic from the data-latch to each SC-DAC input. In this way, it is possible to close the DFE tap-1 timing with reasonable margin, since a conversion step to static CMOS logic is avoided.

Each lane includes an additional offset-programmable latch (amplitude path), shown in Fig. 18 (top), for RX internal eye measurement and DFE tap calibration. It consists of a DCVS latch with integrated voltage offset followed by a set-reset latch. The amplitude bit is fed into the digital calibration block, where the information is processed to find a correlation between the received amplitude samples and previous data bits indicating the presence of ISI or FEXT.

\section{Measurement Results}

The layout of the fabricated circuit, whose RX macro measures $300 \times 350 \mu \mathrm{m}^{2}$, is shown in Fig. 19. The chip, fabricated 


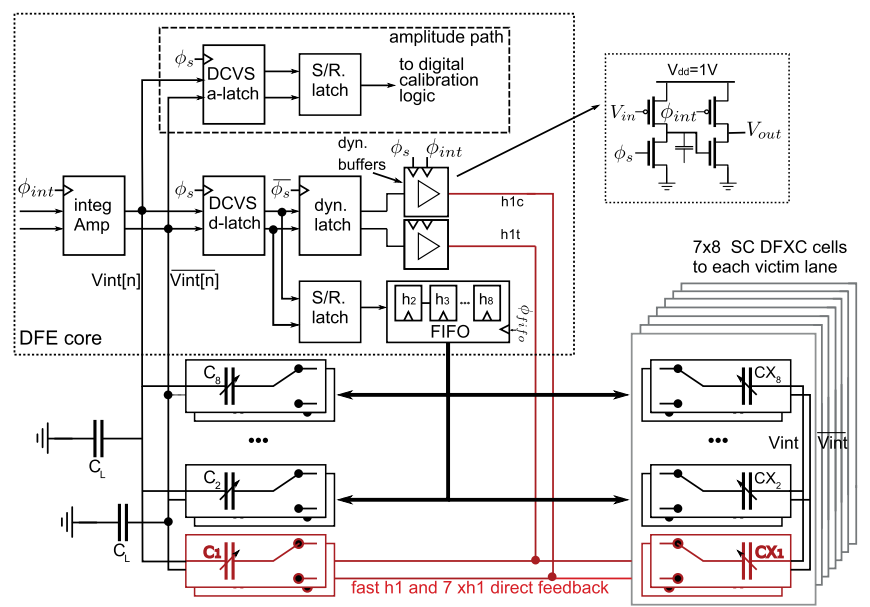

Fig. 18. DFE and DFXC core, with fast tap-1 feedback, including 8-tap DFE and $7 \times 8$ DFXC SC cells.

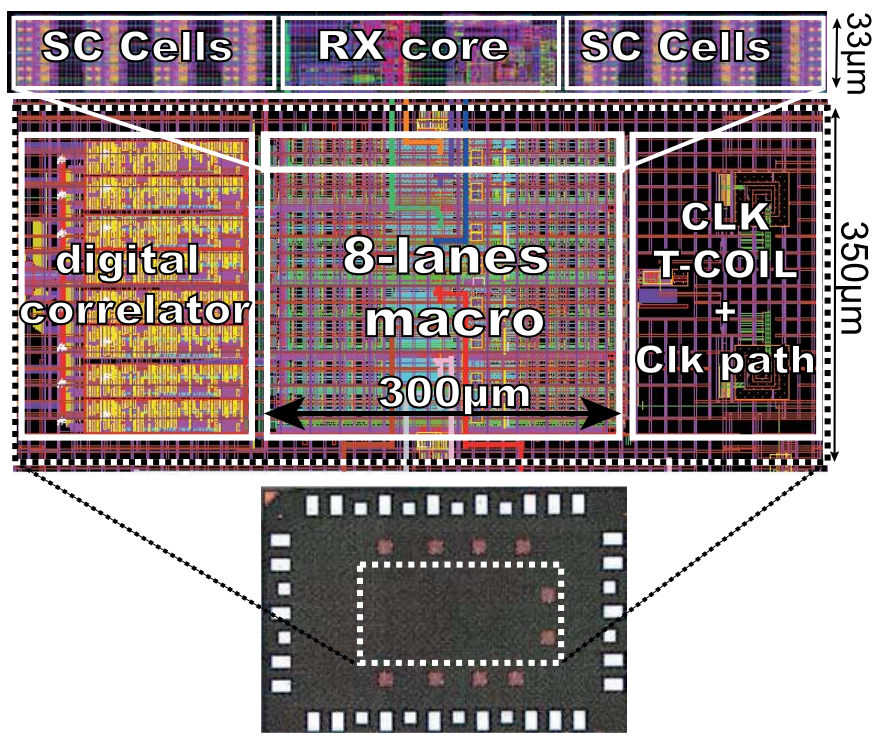

Fig. 19. Layout of RX macro (center), detail of the SC-DFE cells (top), and the die micrograph (bottom).

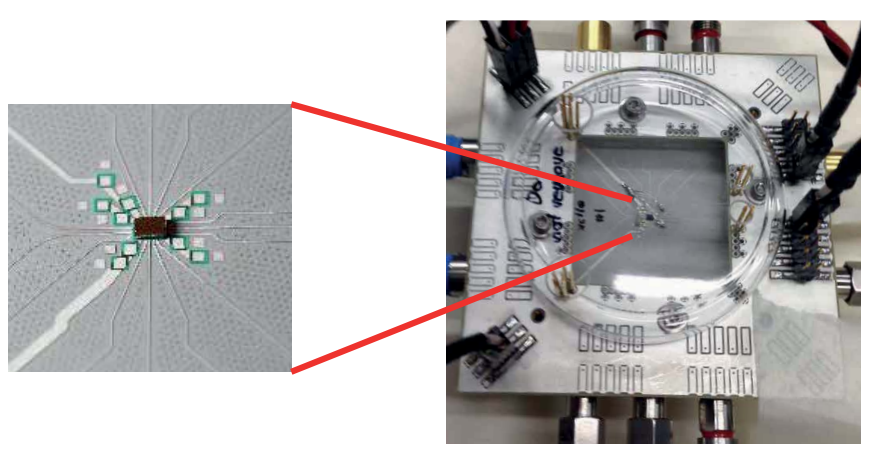

Fig. 20. Left: chip is flip-chip mounted on the LCP PCB. Right: LCP is packaged in a rigid metallic frame.

in 32-nm SOI CMOS, has been flip-chip mounted on a highfrequency, low-loss substrate, liquid crystal polymer (LCP) PCB, shown in Fig. 20 (left). The LCP itself is embedded in a rigid metallic frame, which includes impedance-matched high-frequency coaxial connectors, as shown in Fig. 20 (right).



Fig. 21. Measurement setup: clock generators on top-left, PARBERT for PRBS generation on bottom-left, test board Ch2 on bottom-right, and the RX in the middle.

The RX performances have been tested with both the channels described in Section II-B. The characterization has been performed using high-frequency probing cables connected to an Agilent PARBERT. Fig. 21 shows the measurement setup. Read/write process has been performed thanks to a bidirectional digital interface, used to interface the RX chip with a PC. An on-chip error counter (PRBS checker) and correlator, running at quarter rate, has been exploited to run the electrical characterization for latch offset correction, timing adjustment, and CTXC-CTLE and DFXC-DFE coefficient tuning. A threelane measurement was performed owing to the limitation of the measurement equipment. The data streams sent over the three adjacent lanes were PRBS7 on aggressors and PRBS11 on the victim, thus uncorrelated bit sequences.

\section{A. Ch1 Measurement Results}

The calibration of the internal registers has been addressed as follows: as first step, we calibrate the forward signal path only, switching off the aggressor TXs. The RX output, read by the on-chip amplitude path, is sent to the correlator and analyzed on a PC, using MATLAB tool. The new CTLE-DFE coefficients are written to the internal registers, in order to reduce the ISI. Following this step, we switch on one of the two nearest TX lanes, and we perform the CTXC parameters' sweep, through the PC. The same process is performed for tuning the other nearest aggressor lane, calibrating the second branch of the CTXC. Once the two CTXC set points have been defined, the forward signal calibration is repeated, to reduce the impact of the CTLE on the reduced crosstalk pulse responses, trading off the CTLE and CTXC contribution.

Fig. 22 shows the measured BER bathtub curves related to board Ch1, generated internally by doing a horizontal sweep of the data through the Agilent PARBERT phase generator (32-step UI). Once the aggressor lanes are transmitting, the 


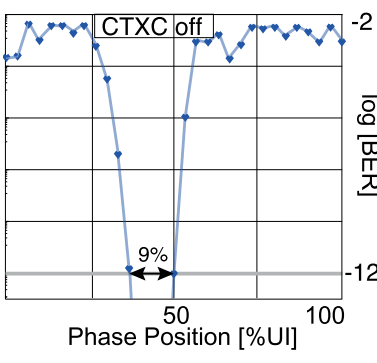

(a)

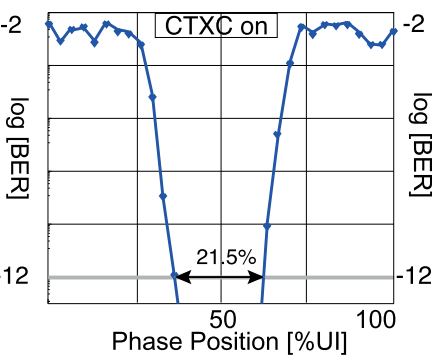

(b)
Fig. 22. Measured bathtub plots for Ch1 board with CTXC (a) switched off and (b) switched on, with the two nearest aggressor lanes transmitting.

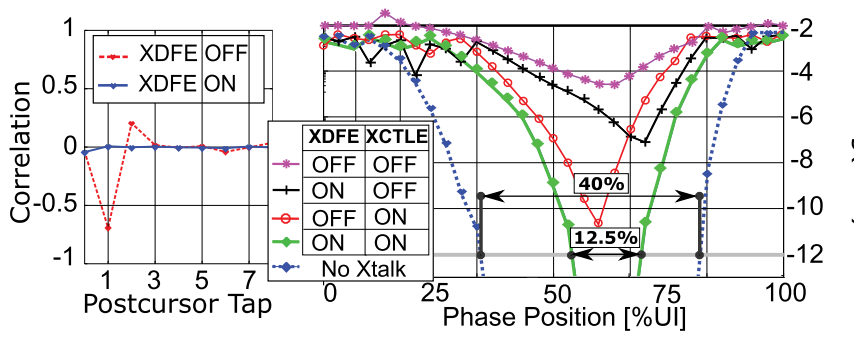

Fig. 23. Board-Ch2. Left: measured correlation with postcursor taps with and without DFXC. Right: measured bathtub plots.

bathtub curve shows a horizontal aperture of $12 \%$ UI, which rises to above $25 \%$ UI, once the CTXC is activated.

\section{B. Ch2 Measurement Results}

The correlation measurement between the two aggressors toward the victim postcursors is necessary for the DFXC taps tuning, over board Ch2. The correlation values were read through the on-chip amplitude path, by the PC and the updated coefficients rewritten to the circuit registers, driving the correlation with postcursor channel taps to zero [see Fig. 23 (left)]. The BER bathtub curves are shown in Fig. 23 (right). With silent aggressors, the RX eye is open with a horizontal margin of $40 \%$ UI at $10^{-12}$ BER. Once the two adjacent aggressor lanes are transmitting, the link does not operate error free, since the bathtub curve reaches only $10^{-4}$ BER. After switching on the crosstalk cancellation blocks, the eye is reasonably open with a $12.5 \%$ UI margin [highlighted in Fig. 24(d)], showing that both CTXC and DFXC are necessary to ensure errorfree operation of the RX. Fig. 24(a)-(c) displays the measured eye diagrams, generated internally by doing a horizontal sweep of the data through the Agilent PARBERT phase generator and vertically by sweeping the amplitude programmable latch offset. The measured vertical eye margins are 22.4 and $64 \mathrm{mV}_{\text {ppdiff }}$ at $10^{-8}$ BER with and without crosstalk, respectively.

A power breakup for 7-Gb/s operation is shown in Table III, which reports the power consumed by one lane. The clock generation circuit is amortized by eight lanes. The DFE core datapath includes integrating amplifier, DCVS latches, dynamic datapath, and digital FIFO. The total power dissipation, once the CTLE, CTXC, 8-tap DFE, and 56-tap DFXC are active, amounts to $5.9 \mathrm{~mW} / \mathrm{Gb} / \mathrm{s}$ with $1-\mathrm{V}$ supply at package,

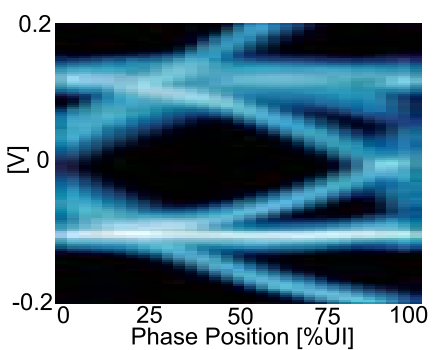

(a)

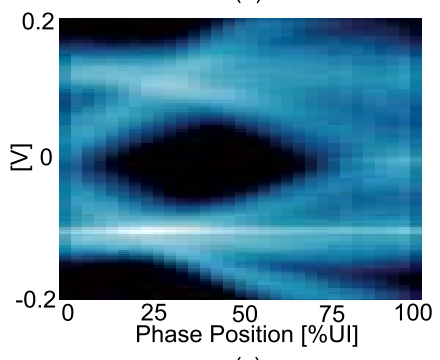

(c)

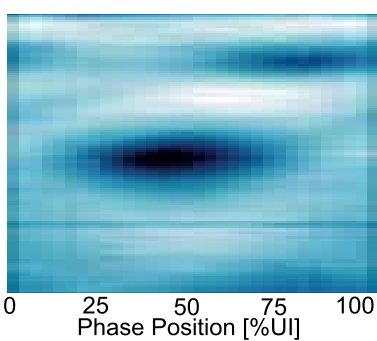

(b)

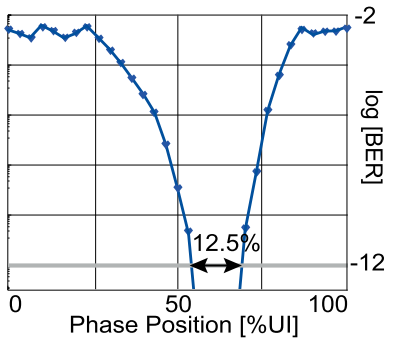

(d)
Fig. 24. Received eye diagrams with silent aggressors (top-left), crosstalk cancellation off (top-right), and crosstalk cancellation activated (bottom-left) with related bathtub plot (bottom-right).

TABLE III

RX POWER DISTRIBUTION

\begin{tabular}{|l|c|}
\hline & $\mu \mathrm{W} / \mathrm{Gb} / \mathrm{s}$ \\
\hline clk path (amortized by 8 lanes) & 66 \\
\hline local clock distribution & 260 \\
\hline CTLE-CTXC & 1150 \\
\hline DFE-DFXC & 3894 \\
\hline digital correlator & 250 \\
\hline 1:4 demux & 280 \\
\hline Total & 5900 \\
\hline
\end{tabular}

from which $3.9 \mathrm{~mW} / \mathrm{Gb} / \mathrm{s}$ are used in the 64-tap DFE+DFXC SC cells and core datapath.

Table IV shows a comparison of the RX macro with prior art. The power overhead compared with the prior art mainly comes from the DFXC function. Moreover, the power number reported here includes the complete RX macro, including digital correlation logic. Finally, the proposed scheme results to be an extremely flexible FEXT compensation scheme, which can be adapted for different single-ended boards.

\section{CONCLUSION}

In this paper, we reported an eight-lane single-ended RX circuit for source-synchronous links for high-loss channels affected by FEXT. Each lane performs ISI equalization and FEXT cancellation based on a CTXC and $7 \times 8$-taps DFXC ensuring robust operation. Unlike the previous literature [13], [16], where crosstalk cancellation schemes were tested on channel with moderate insertion loss, the proposed RX macro can equalize both a 30-dB insertion loss single-ended channel with a signal-to-crosstalk ratio of $0 \mathrm{~dB}$ from the nearest lanes at Nyquist, and a channel with $28 \mathrm{~dB}$ attenuation and reflections due to via stubs with the signal-to-crosstalk ratio of $6 \mathrm{~dB}$. 
TABLE IV

COMPARISON OF EIGHT LANES $\times$ 7-Gb/s RX MACRO With PRIOR ART

\begin{tabular}{|c|c|c|c|c|c|c|}
\hline Reference & [16] & [13] & [20] & [14] & \multicolumn{2}{|c|}{ This work } \\
\hline XTC type & CTXC & Rx passive SC & TX FIR & CTXC & \multicolumn{2}{|c|}{ CTXC, 7x8 DFXC } \\
\hline I/O type & Single-ended & Differential & Single-ended & Single-ended & \multicolumn{2}{|c|}{ Single-ended } \\
\hline Multi-channel num. & 2 & 2 & 2 & 4 & \multicolumn{2}{|c|}{8} \\
\hline Data-rate $(\mathrm{Gb} / \mathrm{s})$ & 6 & 15 & 7 & 12 & \multicolumn{2}{|c|}{7} \\
\hline Channel Attenuation & $9 \mathrm{~dB}$ & $14.5 \mathrm{~dB}$ & N/A & $11 \mathrm{~dB}$ & $30 \mathrm{~dB}$ & $28 \mathrm{~dB}$ \\
\hline Signal-to-Crosstalk ratio & N/A & $1 \mathrm{~dB}$ & N/A & $0 \mathrm{~dB}$ & $0 \mathrm{~dB}$ & $6 \mathrm{~dB}$ \\
\hline Eq. power (pJ/bit/lane) & 2.4 & 0.033 & N/A & 0.96 & \multicolumn{2}{|c|}{8 (full RX) } \\
\hline process node & $130 \mathrm{~nm}$ & $45 \mathrm{~nm} \mathrm{SOI}$ & $40 \mathrm{~nm}$ & $65 \mathrm{~nm}$ & \multicolumn{2}{|c|}{$32 \mathrm{~nm}$ SOI } \\
\hline area $\left(\mathrm{mm}^{2} /\right.$ lane $)$ & 0.03 & N/A & N/A & 0.036 & \multicolumn{2}{|c|}{0.012} \\
\hline
\end{tabular}

The crosstalk reduction strategy can be used across a variety of channels with different crosstalk patterns, due to board geometry. This trend demonstrated with measurements showed good agreement with a system-level analysis. Interestingly, it has been shown how the vertical eye opening improves by increasing CTLE peaking even with severe crosstalk. Moreover, it has been demonstrated that the first 2-4 DFXC taps are sufficient to reduce the crosstalk even in the presence of skew between lanes in the channel bundle.

\section{REFERENCES}

[1] F. O'Mahony, "ISSCC 2017 trends," in Proc. IEEE Int. Solid-States Circuits Conf. Tech Trends, Feb. 2017, p. 2.

[2] ITRS Team, "International technology roadmap for semiconductors," Semiconduct. Ind. Assoc., Washington, DC, USA, Tech. Rep., 2005.

[3] V. Stojanović, "Channel-limited high-speed links: Modeling, analysis and design," Ph.D. dissertation, Stanford Univ. Stanford, CA, USA, 2004.

[4] QuickPath Interconnect, Intel, Santa Clara, CA, USA, Jan. 2009.

[5] Hyper-Transport Link Specification, Rev. 3.10c, document HTC20051222-0046-0003, Hyper Transport Technology Consortium, Sunnyvale, CA, USA, 2010.

[6] S.-K. Lee, B. Kim, H.-J. Park, and J.-Y. Sim, "A 5 Gb/s single-ended parallel receiver with adaptive crosstalk-induced jitter cancellation," IEEE J. Solid-State Circuits, vol. 48, no. 9, pp. 2118-2127, Sep. 2013.

[7] W. Guggenbühl and G. Morbach, "Forward crosstalk compensation on bus lines," IEEE Trans. Circuits Syst. I, Fundam. Theory Appl., vol. 40, no. 8, pp. 523-527, Aug. 1993.

[8] K. Lee, H. K. Jung, H. J. Chi, H. J. Kwon, J. Y. Sim, and H. J. Park, "Serpentine microstrip lines with zero far-end crosstalk for parallel highspeed DRAM interfaces," IEEE Trans. Adv. Packag., vol. 33, no. 2, pp. 552-558, May 2010.

[9] J. F. Buckwalter and A. Hajimiri, "Cancellation of crosstalk-induced jitter," IEEE J. Solid-State Circuits, vol. 41, no. 3, pp. 621-632, Mar. 2006.

[10] K.-I. Oh, L.-S. Kim, K.-I. Park, Y.-H. Jun, J. S. Choi, and K. Kim, "A 5-Gb/s/pin transceiver for DDR memory interface with a crosstalk suppression scheme," IEEE J. Solid-State Circuits, vol. 44, no. 8, pp. 2222-2232, Aug. 2009.

[11] S.-J. Bae et al., "An $80 \mathrm{~nm} 4 \mathrm{~Gb} / \mathrm{s} / \mathrm{pin} 32$ bit $512 \mathrm{Mb}$ GDDR4 graphics DRAM with low power and low noise data bus inversion," IEEE J. Solid-State Circuits, vol. 43, no. 1, pp. 121-131, Jan. 2008.

[12] K.-J. Sham, M. R. Ahmadi, S. B. G. Talbot, and R. Harjani, "FEXT crosstalk cancellation for high-speed serial link design," in Proc. IEEE Custom Integr. Circuits Conf. (CICC), Sep. 2006, pp. 405-408.

[13] M. H. Nazari and A. Emami-Neyestanak, "A 15-Gb/s 0.5-mW/Gbps two-tap DFE receiver with far-end crosstalk cancellation," IEEE J. Solid-State Circuits, vol. 47, no. 10, pp. 2420-2432, Oct. 2012.

[14] T. Oh and R. Harjani, "A 12-Gb/s multichannel I/O using MIMO crosstalk cancellation and signal reutilization in 65-nm CMOS," IEEE J. Solid-State Circuits, vol. 48, no. 6, pp. 1383-1397, Jun. 2013.

[15] A. Cevrero et al., "A $5.9 \mathrm{~mW} / \mathrm{Gb} / \mathrm{s} 7 \mathrm{~Gb} / \mathrm{s} /$ pin 8-lane single-ended RX with crosstalk cancellation scheme using a XCTLE and 56-tap XDFE in $32 \mathrm{~nm}$ SOI CMOS," in Proc. Symp. VLSI Circuits, Jun. 2015, pp. C228-C229.

[16] T. Oh and R. Harjani, "A 6-Gb/s MIMO crosstalk cancellation scheme for high-speed I/Os," IEEE J. Solid-State Circuits, vol. 46, no. 8, pp. 1843-1856, Aug. 2011.
[17] A. Cevrero, "Advanced CMOS circuits for multi-Gb/s links and 3D I/O based on through silicon via technology," Ph.D. dissertation, EPFL, Lausanne, Switzerland, 2014.

[18] T. Toifl et al., "A $2.6 \mathrm{~mW} / \mathrm{Gbps} 12.5$ Gbps RX with 8-tap switchedcapacitor DFE in $32 \mathrm{~nm}$ CMOS," IEEE J. Solid-State Circuits, vol. 47, no. 4, pp. 897-910, Apr. 2012.

[19] M. Kossel et al., "A T-coil-enhanced $8.5 \mathrm{~Gb} / \mathrm{s}$ high-swing SST transmitter in $65 \mathrm{~nm}$ bulk CMOS with $\ll-16 \mathrm{~dB}$ return loss over $10 \mathrm{GHz}$ bandwidth," IEEE J. Solid-State Circuits, vol. 43, no. 12, pp. 2905-2920, Dec. 2008.

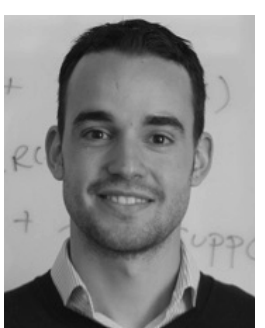

Cosimo Aprile (S'16) received the B.S. degree in electronic engineering from the Politecnico di Torino (POLITO), Turin, Italy, in 2010, and the joint M.S. degree in micro and nanotechnologies for ICTs from the Ecole Polytechnique Fédérale de Lausanne (EPFL), the Institut National Polytechnique de Grenoble (INPG), Grenoble, France, and the Politecnico di Torino, Turin (POLITO). He is currently pursuing the Ph.D. degree in electrical engineering with EPFL.

Since 2013, he has been with the Laboratory for Information and Inference Systems and the Microelectronic Systems Laboratory, EPFL. His current research interests include high-speed analog circuit design, signal processing, and analog/mixed signal integrated circuits design for optimized information extraction from signals or data volumes.

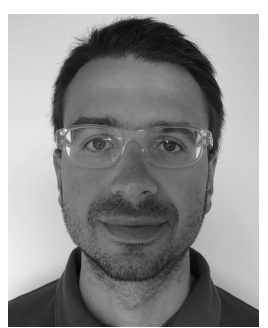

Alessandro Cevrero (M'16) received the M.Sc. degree in nanotechnology and the Ph.D. degree in electrical engineering from the École Polytechnique Fédérale de Lausanne, Lausanne, Switzerland, in 2007 and 2014, respectively.

He joined the IBM Research Zurich, Rüschlikon, Switzerland, in 2012, where he was involved in analog circuit design and silicon validation of highspeed energy-efficient I/O links in advanced CMOS technologies. He has authored or co-authored over 35 technical publications. His current research interests include high-speed analog circuit design, 3-D integration, and semiconductor manufacturing.

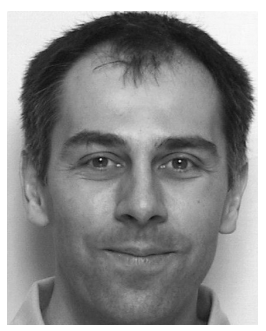

Pier Andrea Francese (M'01-SM'17) received the $\mathrm{Ph} . \mathrm{D}$. degree (cum laude) in electrical engineering from the Politecnico di Milano, Milan, Italy, in 1993, and the Ph.D. degree from ETH Zürich, Zürich, Switzerland, in 2005 .

He was involved in the field of IC product development for Teradyne, Philips Semiconductors, and National Semiconductor. In 2010, he joined the IBM Zurich Research, Rüschlikon, Switzerland, where he develops circuits for energy-efficient high-speed I/O links in advanced CMOS technologies. 


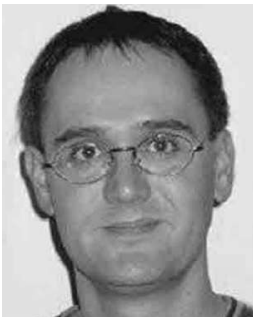

Christian Menolfi (S'97-M'99) received the Dipl.Ing. and the Ph.D. degrees in electrical engineering from ETH Zürich, Zürich, Switzerland, in 1993 and 2000 , respectively.

From 1993 to 2000, he was with the Integrated Systems Laboratory, ETH Zürich, as a Research Assistant, where he was involved in highly sensitive CMOS very-large-scale integration data-acquisition circuits for silicon-based microsensors. Since 2000, he has been with the IBM Research Zurich, Rüschlikon, Switzerland, where he is involved in the design of multigigabit low-power communication circuits in advanced CMOS technologies.

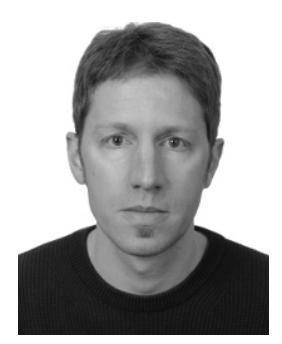

Matthias Braendli received the Dipl.-Ing. (M.Sc.) degree in electrical engineering from ETH Zürich, Zürich, Switzerland, in 1997.

From 1998 to 2001, he was with the Integrated Systems Laboratory, ETH Zürich, where he was involved in deep-submicrometer technology verylarge-scale integration design challenges, digital video image processing for biomedical applications, and testability of CMOS circuits. In 2001, he joined the Microelectronics Design Center, ETH Zürich, where he was involved in numerous digital and mixed-signal application-specific integrated circuit design projects, electronic design automation, and contributed to teaching. In 2008, he joined the IBM Zurich Research, Rüschlikon, Switzerland, where he was involved in multigigabit per second, low-power communication circuits in advanced CMOS technologies.

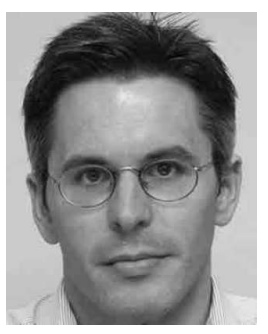

Marcel Kossel (S'99-M'02-SM'09) received the Dipl.-Ing. and $\mathrm{Ph} . \mathrm{D}$. degrees in electrical engineering from ETH Zürich, Zürich, Switzerland, in 1997 and 2000 , respectively.

He joined the IBM Research Zurich, Rüschlikon, Switzerland, in 2001, where he is involved in analog circuit design for high-speed serial links. He has involved in the field of microwave tagging systems and radio frequency identification systems. His current research interests include analog circuit design and radio frequency measurement techniques.

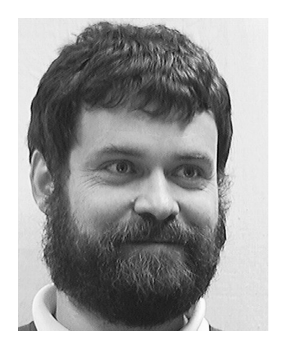

Thomas Morf (S'89-M'90-SM'09) received the B.S. degree from the Zurich University of Applied Science, Winterthur, Switzerland, in 1987, the M.S. degree in electrical and computer engineering from the University of California, Santa Barbara, CA, USA, in 1991, and the Ph.D. degree from ETH Zürich, Switzerland, in 1996.

From 1996 to 1999 , he led a research group in the area of InP-HBT circuit design and technology at ETH. In 1999, he joined the IBM Research Zurich, Rüschlikon, Switzerland. He has co-authored over 150 papers and is a co-inventor of over 30 issued patents. His current research interests include ESD circuit protection, electrical and optical high-speed highdensity interconnects, and terahertz antennas and detectors.

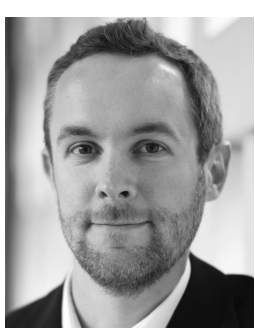

Lukas Kull (S'10-M'14-SM'17) received the M.Sc. degree in electrical engineering from ETH Zürich, Zürich, Switzerland, in 2007, and the Ph.D. degree from the École Polytechnique Fédérale de Lausanne (EPFL), Lausanne, Switzerland, in 2014.

He joined the IBM Research Zurich, Rüschlikon, Switzerland, in 2010, where he has been involved in analog circuit design for high-speed low-power ADCs. He has authored or co-authored over 20 patents and 40 technical publications. His current research interests include analog circuit design, hardware for cognitive workloads, IR, and terahertz imaging.

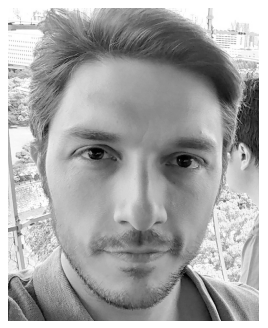

Ilter Oezkaya (S'16) received the B.Sc. degree in electronics engineering from Middle East Technical University, Ankara, Turkey, in 2007, and the M.Sc. degree in electronics engineering from Istanbul Technical University, Istanbul, Turkey, in 2010. He is currently pursuing the Ph.D. degree with the École Polytechnique Fédérale de Lausanne, Lausanne, Switzerland.

He joined the IBM Research Zurich, Rüschlikon, Switzerland, in 2014, where he has been conducting research on analog circuit design for high-speed IO links. His current research interests include high-speed optical and electrical communications and mixed-signal circuit design.

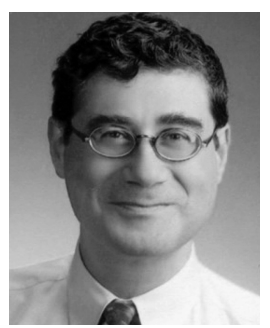

Yusuf Leblebici (M'90-SM'98-F'10) received the B.Sc. and M.Sc. degrees in electrical engineering from Istanbul Technical University, Istanbul, Turkey, in 1984 and 1986, respectively, and the Ph.D. degree in electrical and computer engineering from the University of Illinois at Urbana-Champaign, Champaign, IL, USA, in 1990.

Since 2002, he has been a Chair Professor with the École Polytechnique Fédérale de Lausanne (EPFL), Lausanne, Switzerland, and the Director of the Microelectronic Systems Laboratory, Lausanne. $\mathrm{He}$ is a co-author of six textbooks, and over 300 articles published in various journals and conferences. His current research interests include the design of high-speed CMOS digital and mixed-signal integrated circuits, computeraided design of very-large-scale integration (VLSI) systems, intelligent sensor interfaces, modeling and simulation of semiconductor devices, and VLSI reliability analysis.

Dr. Leblebici was elected as a Distinguished Lecturer of the IEEE Circuits and Systems Society in 2010 and 2011. He has served as an Associate Editor for the IEEE TRANSACTIONS ON CIRCUITS AND SYSTEMS II and the IEEE TRANSACTIONS ON VERY LARGE SCALE INTEGRATED Systems.

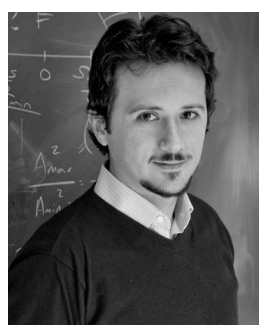

Volkan Cevher (S'95-M'06-SM'10) received the B.Sc. (valedictorian) degree in electrical engineering from Bilkent University, Ankara, Turkey, in 1999, and the $\mathrm{Ph} . \mathrm{D}$. degree in electrical and computer engineering from the Georgia Institute of Technology, Atlanta, GA, USA, in 2005.

He was a Research Scientist with the University of Maryland at College Park, College Park, MD, USA, from 2006 to 2007, and Rice University, Houston, TX, USA, from 2008 to 2009 . He is currently an Associate Professor with the École Polytechnique Fédérale de Lausanne, Lausanne, Switzerland, and a Faculty Fellow with the Electrical and Computer Engineering Department, Rice University. His current research interests include signal processing theory, machine learning, convex optimization, and information theory.

Dr. Cevher was a recipient of the IEEE Signal Processing Society Best Paper Award in 2016, the Best Paper Award at CAMSAP in 2015, the Best Paper Award at SPARS in 2009, and the ERC CG in 2016 and the ERC StG in 2011.

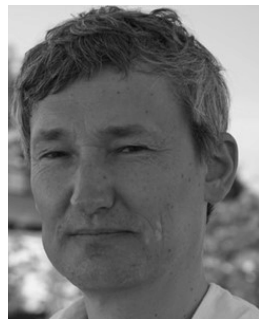

Thomas Toifl (S'97-M'99-SM'09) received the Dipl.-Ing. (M.S.) and Ph.D. (Hons.) degrees in electrical engineering from the Vienna University of Technology, Vienna, Austria, in 1995 and 1999, respectively.

In 1996, he joined the European Research Center for Particle Physics, Microelectronics Group, Geneva, Switzerland, where he developed radiationhard circuits for detector synchronization and data transmission, which were integrated in the four particle detector systems of the new Large Hadron Collider. In 2001, he joined the IBM Zurich Research, Rüschlikon, Switzerland, where he was focusing on multigigabit per second, low-power communication circuits in advanced CMOS technologies. Since 2008, he has been with the I/O Link Technology Group, IBM Zurich Research Laboratory. He has authored or co-authored 19 and over 50 technical publications.

Dr. Toifl received the Beatrice Winner Award for Editorial Excellence at the 2005 IEEE International Solid-State Circuits Conference. 